Pacific Journal of Mathematics

ASYMPTOTIC LOWER BOUNDS FOR THE FREQUENCIES OF 


\title{
ASYMPTOTIC LOWER BOUNDS FOR THE FREQUENCIES OF CERTAIN POLYGONAL MEMBRANES
}

\author{
George E. Forsythe
}

1. Background. Let the bounded, simply connected, open region $R$ of the $(x, y)$ plane have the boundary curve $C$. If a uniform elastic membrane of unit density is uniformly stretched upon $C$ with unit tension across each unit length, the square $\lambda=\lambda(R)$ of the fundamental frequency satisfies the conditions ( subscripts denote differentiation)

$$
\left\{\begin{aligned}
\Delta u & \equiv u_{x x}+u_{y y}=-\lambda u \text { in } R, \\
\lambda & =\text { minimum },
\end{aligned}\right.
$$

with the boundary condition

$$
u(x, y)=0 \text { on } C \text {. }
$$

The solution $u$ of problem ( 1 ) is unique up to a constant factor. It is known $[13, \mathrm{p} .24]$ that $\lambda$ is the minimum over all piecewise smooth functions $u$ satisfying ( $1 \mathrm{~b}$ ) of the Rayleigh quotient

$$
\rho(u)=\iint_{R}|\nabla u|^{2} d x d y / \iint_{R} u^{2} d x d y,
$$

where $|\nabla u|^{2}=u_{x}^{2}+u_{y}^{2}$. In many practical methods for approximating $\lambda$ one essentially determines $\rho(u)$ for functions $u$ satisfying (lb) which are close to a solution of the boundary value problem (1). See $[9$, p. 112;6, p. 276;11, and 12]. By (2) these approximations are known to be upper bounds for $\lambda$; they can be made arbitrarily good with sufficient labor. It is obviously of equal importance to obtain close lower bounds for $\lambda$; cf. [14].

The lower bounds for $\lambda$ given by Pólya and Szegö [13] are ordinarily far

Presented to the American Mathematical Society May 2, 1953, under a slightly different title; received by the editors May 15, 1953. The work on this report was sponsored in part by the Office of Naval Research, USN.

Pacific J. Math. 4 (1954), 467-480 
from close. Those obtainable from $\rho(u), \iint_{R} u^{2} d x d y$, and $\iint_{R}|\Delta u|^{2} d x d y$ by methods due to Temple [15], D. H. Weinstein [17], Wielandt [18], and Kato [8] (for expositions see [3] and [16]) are arbitrarily good, but presuppose knowledge of a lower bound for the second eigenvalue $\lambda_{2}$ of the problem (1). The same is true of Davis's proposals in [4]. It is possible, following Aronszajn and Zeichner [1], to get close lower bounds for $\lambda$ by minimizing $\rho(u)$ over a class of functions $u$ permitted some discontinuity in $R$ (method of A. Weinstein); the author has no knowledge of the practicability of the method.

A common method of approximating $\lambda$ is to replace the boundary value problem (1) by a similar problem in finite differences. Divide the plane into squares of side $h$ by the network of lines $x=\mu h, y=\nu h(\mu, \nu=0, \pm 1, \pm 2, \cdots)$. The points $(\mu h, \nu h)$ are the nodes of the net. A half-square is an isosceles right triangle whose vertices are three nodes of one square of the net. Assume that

$R$ is the union of a finite number of squares and half-squares.

Then every interior node of $R$ has four neighboring nodes in $R \cup C$.

Define $\Delta_{h}$, a finite-difference approximation to $\Delta$, by the relation

$$
h^{2} \Delta_{h} v(x, y)=v(x+h, y)+v(x-h, y)+v(x, y+h)+v(x, y-h)-4 v(x, y) .
$$

Let $\lambda_{h}$ be the least number satisfying the following difference equation for a net function $v$ defined on the nodes $(x, y)$ of the net:

$$
\Delta_{h} v=-\lambda_{h} v \text { at the nodes in } R \text {, }
$$

with the boundary condition

$$
v=0 \text { at the nodes on } C \text {. }
$$

One can interpret $\lambda_{h}$ as the square of the fundamental frequency of a network of massless strings with uniform tension $h$, fastened to $C$, and supporting a particle of mass $h^{2}$ at each node. That is, a certain lumping of the distributed masses and tensions of problem (1) yields problem (4).

It is easily verified for a rectangular region of commensurable sides $\pi / p$, $\pi / q$, and for $h$ such that (3) holds, that one has $u=v=\sin p x \sin q y$, and that

$$
\frac{\lambda_{h}}{\lambda}=\frac{\sin ^{2}(p h / 2)+\sin ^{2}(q h / 2)}{(p h / 2)^{2}+(q h / 2)^{2}}=1-\frac{p^{4}+q^{4}}{p^{2}+q^{2}} \frac{h^{2}}{12}+o\left(h^{2}\right) \quad(h \rightarrow 0) .
$$

Hence $\lambda_{h}<\lambda$ for all $h$, and one can use $\lambda_{h}$ as a lower bound for $\lambda$. However, 
since $\lambda$ is known exactly for rectangular regions, relation (5) contributes nothing to its computation. For general regions $R$, it was stated [3, p.405] in 1949 that nothing could be said about the relation of $\lambda_{h}$ to $\lambda$.

2. A new result. An asymptotic relation resembling (5) will now be established for any convex polygonal region $R$ satisfying (3). Such regions are polygons of at most eight sides, having interior vertex angles of $45^{\circ}, 90^{\circ}$, or $135^{\circ}$. The following theorem ${ }^{1}$ will be proved in $\S 3$ by use of the lemmas of $\S 4$ :

THEOREM. Let $R$ be a convex region which is a finite union of squares and half-squares for all $h$ under consideration. Let u solve problem (1) for $R$, and let

$$
a=a(R)=\frac{\iint_{R}\left(u_{x x}^{2}+u_{y y}^{2}\right) d x d y}{\iint_{R}\left(u_{x}^{2}+u_{y}^{2}\right) d x d y} .
$$

Then, as $h \longrightarrow 0$, one has

$$
\frac{\lambda_{h}}{\lambda} \leq 1-\frac{a}{12} h^{2}+o\left(h^{2}\right)
$$

It is a consequence of the theorem that, for all sufficiently small $h$, say for $h \leq h_{0}, \lambda_{h}$ is a lower bound for $\lambda$. The ordinary finite-difference method thus complements any method based on Rayleigh quotients; and, since $\lambda_{h} \rightarrow \lambda$ as $h \longrightarrow 0$, together two such methods can confine $\lambda$ to an arbitrarily short interval. In particular, Pólya [11 and 12] devises modified finite-difference approximations to problem (1) which furnish upper bounds to $\lambda$ for all $h$. Hence arbitrarily good two-sided bounds to $\lambda$ can be found by finite-difference methods alone.

The constant $a$ of the theorem is the best possible for a rectangle $R$ of sides $\pi / p, \pi / q$. For this region, we have $a=\left(p^{4}+q^{4}\right) \cdot\left(p^{2}+q^{2}\right)^{-1}$, and (6) is seen by $(5)$ to be actually an equality up to terms $o\left(h^{2}\right)$.

Using heuristic reasoning, Milne $[9$, p. 238, (97.5)] finds an approximate formula which, specialized to the fundamental eigenvalue and set in our notation, says

$$
\frac{\lambda_{h}}{\lambda} \doteq 1-\frac{\lambda h^{2}}{24}+o\left(h^{2}\right)
$$

1 The author gratefully acknowledges many helpful conversations with his colleague Dr. Wolfgang Wasow on the subject of this paper. 
For a rectangle of sides $\pi / p, \pi / q$, the coefficient of $-h^{2} / 12$ in $(7)$ is $\left(p^{2}+q^{2}\right) / 2$. Since

$$
\frac{p^{2}+q^{2}}{2}+\frac{\left(p^{2}-q^{2}\right)^{2}}{p^{2}+q^{2}}=\frac{p^{4}+q^{4}}{p^{2}+q^{2}},
$$

the coefficient of $h^{2}$ in (7) is low for all rectangles with $p \neq q$, and exact for squares. Hence (7) cannot ordinarily be expected to be exact in its $h^{2}$ term.

The use of the theorem to bound $\lambda$ is limited by our lack of knowledge of $h_{0}$. However, it is the author's conjecture that, for the regions $R$ of the theorem, $\lambda_{h}<\lambda$ for all $h$.

The convexity of $R$ is vital to the statement and proof of the theorem; in fact, by the remark after Lemma $4, a=\infty$ for nonconvex polygons. A heuristic argument, supported by the numerical example of $\xi 5$, has in fact convinced the author that, for nonconvex polygons, $\lambda_{h}>\lambda$ for all sufficiently small $h$.

The restriction of $R$ and $h$ to satisfy (3) is less essential, but is used in two ways: (i) to be sure that no interior node has a neighboring node outside $R$; (ii) to prove that $\Gamma=0$ in Lemma 7 . With an appropriate alteration of $\Delta_{h}$ near $C$, and with a modification of Lemma 7 , one can extend the present method to obtain formulas of type (6) without assuming (3)-and even for convex regions $R$ bounded by piecewise analytic curves $C$. See [5]. Analogous results can be expected in $n$ dimensions.

3. Proof of the theorem. Let $K$ be the class of functions $u$ which vanish on $C$, such that $\left(u u_{x}\right)_{x}$ and $\left(u u_{y}\right)_{y}$ are continuous in $R \cup C$. Applying Gauss's divergence formula (27) with $p=u u_{x}, q=u u_{y}$, one finds that, for all $u$ in $K$, Green's formula is valid in the form

$$
\iint_{R}|\nabla u|^{2} d x d y=-\iint_{R} u \Delta u d x d y .
$$

Hence, for all $u \in K, \rho(u)$ in (2) can be rewritten with $-\iint_{R} u \Delta u d x d y$ in the numerator.

Since, by Lemma 1 , the function $u$ which minimizes (2) and solves (1) belongs to $K$, and since any function in $K$ is piecewise smooth, one may alternatively define $\lambda$ as the minimum, over all functions in $K$, of the quotient

$$
\rho(u)=-\iint_{R} u \Delta u d x d y / \iint_{R} u^{2} d x d y .
$$


Analogously, without having to worry about function classes, one can show that $\lambda_{h}$ is the minimum, over all net functions $v$ satisfying (4b), of the quotient

$$
\rho_{h}(v)=-h^{2} \sum \sum_{N_{h}} v \Delta_{h} v / h^{2} \sum_{N_{h}} \sum^{2}
$$

where the sums are extended over all nodes $N_{h}$ of the net inside $R$.

The key to proving the theorem is to set the solution $u$ of problem (1) into the Rayleigh quotient (8) of problem (4). It will be shown that

$$
\frac{\rho_{h}(u)}{\lambda}=1-\frac{1}{12} a h^{2}+o\left(h^{2}\right)
$$

Since $\lambda_{h} \leq \rho_{h}(u)$, the theorem follows from (9). Henceforth $u$ will always denote a solution of problem (1).

The denominator of $\rho_{h}(u)$ is a Riemann sum for $\iint_{R} u^{2} d x d y$. Since $u^{2}$ is continuous and hence Riemann integrable over $R$,

$$
h^{2} \sum \sum_{N_{h}} u^{2}=\iint_{R} u^{2} d x d y+o(1)
$$

(It can be shown that one can replace $o(1)$ by $o\left(h^{2}\right)$ in (10), but we shall not need to do this.)

The nodes $N_{h}$ inside $R$ are divided into two classes:

$$
\begin{aligned}
& N_{h}^{\prime} \text { : those at a distance } h \text { from some } 135^{\circ} \text { vertex of } C \text {; } \\
& N_{h}^{\prime \prime} \text { : the other nodes of } N_{h} \text {. }
\end{aligned}
$$

Split the numerator of $\rho_{h}(u)$ accordingly:

$$
-h^{2} \sum_{N_{h}} \sum_{N_{h}^{\prime}} u \Delta_{h} u=-h^{2} \sum_{N_{h^{\prime \prime}}} u \Delta_{h} u-h^{2} \sum_{N^{\prime \prime}} u \Delta_{h} u=S_{h}^{\prime}(u)+S_{h}^{\prime \prime}(u)
$$

To estimate $S_{h}^{\prime}(u)$ note that, since there are at most eight $135^{\circ}$ vertices, the number of nodes in $N_{h}^{\prime}$ is at most 8 , for any $h$. At any node in $N_{h}^{\prime}$,

$$
h^{2}\left|u \Delta_{h} u\right| \leq h^{2}\left(\frac{u-0}{h}\right) \sum_{i=1}^{4}\left|\frac{u-u_{i}}{h}\right| \leq 4 h^{2} \max |\nabla u|^{2},
$$


where the maximum of $|\nabla u|^{2}$ is taken for all points $(x, y)$ within a distance $2 h$ of some $135^{\circ}$ vertex. Hience, by Lemma 2 , as $h \rightarrow 0$ through values such that (3) holds,

$$
\left|S_{h}^{\prime}(u)\right| \leq 32 h^{2} \max |\nabla u|^{2}=o\left(h^{2}\right) \quad(h \rightarrow 0) .
$$

Now, using the notation and assertion of Lemma 5, one obtains

$$
S_{h}^{\prime \prime}(u)=-h^{2} \sum_{N_{h}^{\prime \prime}} u \Delta u-\frac{h^{4}}{12} \sum_{N_{h}^{\prime \prime}} \sum_{x} u\left(u_{x x x x}^{\prime}+u_{y y y y}^{\prime \prime}\right)
$$

Since $u$ satisfies (la),

$$
-h^{2} \sum_{N_{h}^{\text {II }}} \sum_{N_{h}^{\text {I’ }}} u \Delta u=\lambda h^{2} \sum_{N_{h}} u^{2}=\lambda h^{2} \sum_{N} u^{2}+o\left(h^{2}\right) \quad(h \rightarrow 0) ;
$$

the last step is correct because $u(x, y) \rightarrow 0$ as $(x, y) \rightarrow C$.

Combining ( 13 ) and (14), one finds that, as $h \rightarrow 0$,

$$
S_{h}^{\prime \prime}(u)=\lambda h^{2} \sum_{N_{h}} \sum_{N_{h}^{\prime \prime}} u^{2} \frac{h^{4}}{12} \sum_{N_{h}^{\prime \prime}} u\left(u_{x x x x}^{\prime}+u_{y y y y}^{\prime \prime}\right)+o\left(h^{2}\right)
$$

(15)

$$
=\lambda h^{2} \sum_{N_{h}} u^{2}-\frac{h^{2}}{12} \iint_{R} u\left(u_{x x x x}+u_{y y y y}\right) d x d y+o\left(h^{2}\right),
$$

by Lemma 6. The integrals used in this proof exist, by Lemma 3. Using (11), (12), (15), and Lemma 7, one finds that

(16) $-h^{2} \sum_{N_{h}} \sum_{h} u \Delta_{h}$

$$
=\lambda h^{2} \sum_{N_{h}} \sum^{2}-\frac{h^{2}}{12} \iint_{R}\left(u_{x x}^{2}+u_{y y}^{2}\right) d x d y+o\left(h^{2}\right) \quad(h \rightarrow 0) .
$$

Dividing ( 16$)$ by the denominator of $\rho_{h}(u)$, one gets 


$$
\rho_{h}(u)=\lambda-\frac{h^{2}}{12} \frac{\iint_{k}\left(u_{x x}^{2}+u_{y y}^{2}\right) d x d y}{h^{2} \sum_{N_{h}} u^{2}}+o\left(h^{2}\right)
$$

Hence, by (10),

$$
\rho_{h}(u)=\lambda-\frac{h^{2}}{12} \frac{\iint_{R}\left(u_{x x}^{2}+u_{y y}^{2}\right) d x d y}{\iint_{R} u^{2} d x d y}+o\left(h^{2}\right) \quad(h \rightarrow 0)
$$

If one divides (17) by $\lambda$, and notes from (2) that $\lambda \iint_{R} u^{2} d x d y=\iint_{R}|\nabla u|^{2} d x d y$, it is seen that

$$
\frac{\rho_{h}(u)}{\lambda}=1-\frac{h^{2}}{12} \frac{\iint_{R}\left(u_{x x}^{2}+u_{y y}^{2}\right) d x d y}{\iint_{R}|\nabla u|^{2} d x d y}+o\left(h^{2}\right) \quad(h \rightarrow 0) .
$$

By the definition of $a$ we have proved (9) and hence the theorem.

4. Some lemmas. Lemma 1, suggested to the author by Professor Max Shiffman, is used to establish Lemmas 2 to 7 , which were applied to prove the theorem. In all the lemmas $R$ is the convex union of squares and half-squares of the network, while $u=u(x, y)$ is a function solving problem (1) in $k$.

LEMMA 1. The function $u$ is an analytic function of $x$ and $y$ in $R \cup C$, except at the $135^{\circ}$ vertices of $C$. Let $r, \theta$ be local polar coordinates centered at a $135^{\circ}$ vertex $P_{k}$, with $0<\theta<3 \pi / 4$ in $R$. Then

$$
u=\gamma_{k} r^{4 / 3} \sin (4 \theta / 3)+r^{7 / 3} E_{k}(r, \theta),
$$

where $\gamma_{k}$ is a constant, and where $E_{k}(r, \theta)$, together with all its derivatives, is bounded in a neighborhood of $P_{k}$.

Proof. By reflection one can continue $u$ antisymmetrically across each straight segment of $C$, and ( $l a$ ) is satisfied by the extended $u$ at all points of $R \cup C$ except the $135^{\circ}$ vertices. The first sentence of the lemma then follows from $[2, \mathrm{p} .179]$.

For $(\xi, \eta) \in R$, write $t=\xi+i \eta$. For each $t$, let $w=f(z, t)$ be an analytic function of the complex variable $z=x+i y$ which maps $R$ into the unit circle $|w|<1$, with $f(t, t)=0$. To study $f$ near a vertex $z_{k}$ of $C$, one may assume 
that $f\left(z_{k}, t\right)=1$. Let the interior vertex angle of $C$ at $z_{k}$ be $\pi / \alpha_{k}\left(\alpha_{k}=4,2\right.$, or $4 / 3)$. It is a property of the Schwarz-Christoffel transformation [10, p. 189] that

$$
f(z, t)=1+\left(z-z_{k}\right)^{\alpha} g_{k}(z, t),
$$

where $g_{k}$ is an analytic function of $z$ regular at $z_{k}$.

Let $G(z, t)=G(x, y ; \xi, \eta)$ be Green's function for $\Delta u$ in $R$. Now $G(z, t)=-$ $(2 \pi)^{-1} \log |f(z, t)| ;$ see [10, p. 181]. It then follows from (19) that, in the notation of the lemma, when $\alpha_{k}=4 / 3$,

$$
G(z, t)=y_{k}(t) r^{4 / 3} \sin (4 \theta / 3)+r^{7 / 3} E_{k}(r, \theta, t) .
$$

Moreover, $\gamma_{k}(t)$ and $E_{k}(r, \theta, t)$ are integrable over $R$, since the only discontinuity of $G(z, t)$ is a logarithmic one at $t=z$.

The function $u$ is representable by the integral [2, pp. 182-3]

$$
u(x, y)=\lambda \iint_{R} G(x, y ; \xi, \eta) u(\xi, \eta) d \xi d \eta
$$

Substituting (20) into (21) proves (18) and the lemma.

Lemma 2. $|\nabla u(x, y)| \rightarrow 0$ as $(x, y) \rightarrow$ any $135^{\circ}$ vertex of $C$.

Proof. By (18), $|\nabla u|=O\left(r^{1 / 3}\right)$, as $(x, y) \rightarrow$ any $135^{\circ}$ vertex of $C$.

LEMmA 3. The functions $u_{x x}^{2}, u_{x} u_{x x x}, u u_{x x x x}, u_{y y}^{2}, u_{y} u_{y y y}$, and $u u_{y y y y}$ are Lebesgue-integrable in $R$.

Proof. By Lemma 1 these functions are continuous in $R \cup C$, except at the $135^{\circ}$ vertices $P_{k}$. At these vertices (18) implies that they are $O\left(r^{-4 / 3}\right)$ and are hence integrable.

Lemma 4. The Lebesgue integrals $\int_{C} u_{y} u_{y y} d x$ and $\int_{C} u_{x} u_{x x} d y$ exist.

Proof. Analogous to that of Lemma 3.

REMARK. Lemmas 2,3 , and 4 are false for polygonal regions $R$ which are not convex, since in general the exponent in (18) is $\alpha_{k}$, where $\pi / \alpha_{k}$ is the interior angle at the vertex $P_{k}$.

Lemma 5. At each node $(x, y)$ in $R$ of the network of section 1 , one has 


$$
\Delta_{h} u=\Delta u+\frac{1}{12} h^{2}\left(u_{x x x x}^{\prime}+u_{y y y y}^{\prime \prime}\right)
$$

where

$$
\left\{\begin{array}{l}
u_{x x x x}^{\prime}=u_{x x x x}\left(x+\theta^{\prime} h, y\right),-1<\theta^{\prime}<1 ; \\
u_{y y y y}^{\prime \prime}=u_{y y y y}\left(x, y+\theta^{\prime \prime} h\right),-1<\theta^{\prime \prime}<1 .
\end{array}\right.
$$

Proof. By Lemma $1, u_{x x x x}$ is continuous in the open line segment from $(x-h, y)$ to $(x+h, y)$ (though infinite at any $135^{\circ}$ vertex). Since $u$ is continuous in $R \cup C$, it follows from Taylor's formula [7, p.357] that, if we fix $y$ and set $\phi(x)=u(x, y)$,

$$
\begin{aligned}
\phi(x+h)+ & \phi(x-h)-2 \phi(x) \\
& =h^{2} \phi^{\prime \prime}(x)+\frac{1}{24} h^{4}\left[\phi^{\prime \prime \prime \prime}\left(x+\theta_{1} h\right)+\theta^{\prime \prime \prime \prime}\left(x-\theta_{2} h\right)\right],
\end{aligned}
$$

where $0<\theta_{i}<1 \quad(i=1,2)$. By the continuity of $\phi^{\prime \prime \prime \prime, ~ t h e ~ l a s t ~ b r a c k e t ~ e q u a l s ~}$ $2 \phi^{\prime \prime \prime \prime}\left(x+\theta^{\prime} h\right)$, where $-1<\theta^{\prime}<1$.

A similar formula for $\psi(y)=u(x, y)$, when added to the above and divided by $h^{2}$, yields (22) and ( 23 ).

Lemma 6. Define $N_{h}^{\prime \prime}$ as in $\$ 3$. For each node $(x, y)$ in $N_{h}^{\prime \prime}$, use the notation of (23). Then, as $h \longrightarrow 0$ over values such that (3) holds, one has

$$
h^{2} \sum_{N_{h}^{\prime \prime}} u\left(u_{x x x x}^{\prime}+u_{y y y y}^{\prime \prime}\right)=\iint_{R} u\left(u_{x x x x}+u_{y y y y}\right) d x d y+o(1) \quad(h \rightarrow 0)
$$

Proof. For all $(x, y)$ in the entire plane $E_{2}$ define

$$
f(x, y)=\left\{\begin{array}{l}
u\left(u_{x x x x}+u_{y y y y}\right), \text { if }(x, y) \in R \\
0, \text { elsewhere }
\end{array}\right.
$$

By the proof of Lemma 3 one sees that $f(x, y)$ is $O\left(r^{-4 / 3}\right)$ in the neighborhood of each $135^{\circ}$ vertex $P_{k}$ of $C$, and continuous elsewhere. Divide the nodes $(x, y)=$ $(\mu h, \nu h)$ of $N_{h}^{\prime \prime} \subset R$ into four classes $K^{(i)}(i=1,2,3,4)$ according to the parity of $(\mu, \nu)$. Fix any class $K^{(i)}$. For each vertex $(x, y)$ in $K^{(i)}$ let $S(x, y)$ be the union of the four closed network squares of $E_{2}$ which contain $(x, y)$. The area 
of each $S(x, y)$ is $4 h^{2}$; ordinarily certain of the $S(x, y)$ contain points not in $R$. Define

$$
f_{h}^{(i)}(\xi, \eta)=\left\{\begin{array}{l}
u(x, y)\left(u_{x x x x}^{\prime}+u_{y y y y}^{\prime \prime}\right), \text { for }(\xi, \eta) \in S(x, y) ; \\
0, \quad \text { for }(\xi, \eta) \notin \cup S(x, y) .
\end{array}\right.
$$

Then $f_{h}^{(i)}(\xi, \eta) \rightarrow f(\xi, \eta)$, as $h \longrightarrow 0$, for almost all $(\xi, \eta)$ in the plane. Using the fact that no node of $N_{h}^{\prime \prime}$ is adjacent to a $135^{\circ}$ vertex of $C$, one can show that for all $i$, uniformly in $h,\left|f_{k}^{(i)}(\xi, \eta)\right| \leq F(\xi, \eta)$, where $F$ is an integrable function in $E_{2}$.

Each term of the sum $(24)$ for which $(x, y) \in K^{(i)}$ is equal to

$$
\frac{1}{4} \iint_{S(x, y)} f_{h}^{(i)}(\xi, \eta) d \xi d \eta
$$

Hence, applying Lebesgue's convergence theorem, one sees that, as $h \rightarrow 0$, for each $i$,

$$
\sum_{N_{h}^{\prime \prime} \cap K} \sum_{(i)} u\left(u_{x x x x}^{\prime}+u_{y y y y}^{\prime \prime}\right)=\frac{1}{4} \iint_{E_{2}} f_{h}^{(i)}(\xi, \eta) d \xi d \eta
$$

$$
\rightarrow \frac{1}{4} \iint_{E_{2}} f(\xi, \eta) d \xi d \eta
$$

Summing ( 25 ) over $i=1,2,3,4$ proves (24) and the lemma.

LEMMA 7. One has

$$
\iint_{R} u\left(u_{x x x x}+u_{y y y y}\right) d x d y=\iint_{R}\left(u_{x x}^{2}+u_{y y}^{2}\right) d x d y .
$$

Proof. The following applications of Gauss's divergence theorem in the form

$$
\iint_{R}\left(p_{x}+q_{y}\right) d x d y=\int_{C}(p d y-q d x)
$$

can be justified by integrating over the region $R^{*}$ interior to a smooth convex curve $C^{*}$ inside $R$, and then letting $C^{*} \longrightarrow C$ appropriately. The continuity of 
the integrals in the limit follows from Lemmas 1,3 , and 4.

In the divergence theorem for $p=u u_{x x x s} q=u u_{y y y}$, the line integral vanishes, and one finds

$$
\iint_{R} u\left(u_{x x x x}+u_{y y y y}\right) d x d y=-\iint_{R}\left(u_{x} u_{x x x}+u_{y} u_{y y y}\right) d x d y
$$

A second application of the divergence theorem with $p=u_{x} u_{x x}, q=u_{y} u_{y y}$, combined with (28), shows that

$$
\iint_{R} u\left(u_{x x x x}+u_{y y y y}\right) d x d y=\iint_{R}\left(u_{x x}^{2}+u_{y y}^{2}\right) d x d y+\Gamma,
$$

where $\Gamma=\int_{C}\left(u_{y} u_{y y} d x-u_{x} u_{x x} d y\right)$.

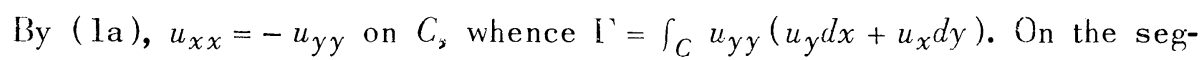
ments of $C$ parallel to the axes, $u_{x x}=u_{y y}=0$, so that there the contribution to $\Gamma$ is zero.

Now the vector $\nabla u=\left(u_{x}, u_{y}\right)$ is perpendicular to $C$. On the segments of $C$ making a $45^{\circ}$ or $135^{\circ}$ angle with the $x$-axis, $\left(u_{y}, u_{x}\right)$ is parallel to $\left(u_{x}, u_{y}\right)$, whence $\left(u_{y}, u_{x}\right)$ is perpendicular to $C$. Thus $u_{y} d x+u_{x} d y \equiv 0$ when $(d x, d y)$ is tangent to $C$, so that the contribution to $\Gamma$ from these $45^{\circ}$ and $135^{\circ}$ segments of $C$ is also zero.

Hence $\Gamma=0$, and the lemma follows from ( 29 ).

5. Numerical example. Let $R_{1}$ be the six-sided, nonconvex, $L$-shaped region whose closure is the union of the three unit squares

$$
\left\{\begin{array}{rlrl}
-1 & \leq x \leq 0, & 0 \leq y \leq 1 ; \\
0 \leq x \leq 1, & 0 \leq y \leq 1 ; \\
0 \leq x \leq 1, & -1 \leq y \leq 0 .
\end{array}\right.
$$

The fundamental frequencies $\lambda_{h}=\lambda_{h}\left(R_{1}\right)$ and corresponding net functions $v$ were computed by B.F. Handy on the SWAC (National Bureau of Standards Niestern Automatic Computer) for $1 / h=3,4, \cdots, 8$. The computation used a power method; for some initial net function $v_{0},\left(h^{2} \Delta_{h}+5 I\right)^{m} v_{0}$ was determined for large positive integers $m$, where $I$ is the identity operator. On the basis of Collatz's inclusion theorem [3, p. 289], the values in the accompanying table are believed to have errors less than $5 \times 10^{-6}$. Observe that $\lambda_{h}\left(R_{1}\right)$ is less for $h=1 / 8$ than for $h=1 / 7$. 
TABLE

$\begin{array}{ccc}h & \lambda_{h}\left(R_{1}\right) & \lambda_{h}\left(R_{2}\right) \\ 1 / 2 & 9.07180 & 12.00000 \\ 1 / 3 & 9.52514 & 13.73700 \\ 1 / 4 & 9.64143 & 14.37340 \\ 1 / 5 & 9.67860 & 14.67081 \\ 1 / 6 & 9.69083 & 14.83259 \\ 1 / 7 & 9.69384 & 14.93003 \\ 1 / 8 & 9.69316 & 14.99315\end{array}$

Since $R_{1}$ is not convex, the theorem of $\S 2$ does not apply, but a heuristic argument suggests that $\lambda_{h}\left(R_{1}\right)-\lambda\left(R_{1}\right)=O\left(h^{4 / 3}\right)$. A least-squares fit to the values of $\lambda_{h}\left(R_{1}\right)$ for $1 / 8 \leq h \leq 1 / 4$ of a function of type

$$
\lambda_{h}\left(R_{1}\right) \doteq \alpha_{1}+\beta_{1} h^{4 / 3}+\gamma_{1} h^{2}=\phi_{1}(h)
$$

yielded the values

$$
\alpha_{1}=9.63632, \quad \beta_{1}=2.40286, \quad \gamma_{1}=-5.97212 \text {. }
$$

The maximum of $\left|\lambda_{h}\left(R_{1}\right)-\phi_{1}(h)\right|$ for the five values of $h$ is .00013 . Hence $\alpha_{1}$ is a working estimate of $\lambda\left(R_{1}\right)$.

The fact that $\beta_{1}>0$ in (30) supports the author's conjecture that, for nonconvex polygonal domains satisfying (3), $\lambda_{h}>\lambda$ for all sufficiently small $h$.

The table also gives Handy's values for the second eigenvalues of $R_{1}$, which are the fundamental eigenvalues $\lambda_{h}\left(R_{2}\right)$ of the trapezoidal halfdomain $R_{2}$ of $R_{1}$ for which $x>y$. Since the theorem does apply to $R_{2}$, a least-squares fit to the values of $\lambda_{h}\left(R_{2}\right)$ for $1 / 8 \leq h \leq 1 / 4$ of a function of type

$$
\lambda_{h}\left(R_{2}\right) \doteq \alpha_{2}+\beta_{2} h^{2}=\phi_{2}(h)
$$

seemed appropriate, and yielded the values

$$
\alpha_{2}=15.19980, \quad \beta_{2}=-13.22219 \text {. }
$$

The maximum of $\left|\lambda_{h}\left(R_{2}\right)-\phi_{2}(h)\right|$ for the five values of $h$ was .00010 . Hence $\alpha_{2}$ is a working estimate of $\lambda\left(R_{2}\right)$.

The value of $\beta_{2}$ is negative, in agreement with (6), but the quantity 
$-12 \beta_{2} / \alpha_{2}=10.4387$ is something like one-fifth larger than an estimate of the corresponding quantity $a\left(R_{2}\right)$ of the theorem. One therefore suspects that $a$ is not the best possible constant in (6) for the region $R_{2}$.

In the table, note the relative closeness of the values of $\lambda_{h}\left(R_{2}\right)$ to the working estimate, $\alpha_{2}$, of $\lambda\left(R_{2}\right)$, even for a coarse net. Thus the value 12 for $\lambda_{1 / 2}\left(R_{2}\right)$, which is obtained by pencil and paper from a simple quadratic equation, is comparable to the lower bounds 12.1 and $5 \pi^{2} / 4$ obtained respectively by comparison with $\lambda$ for the circular membrane of equal area $[13$, p. 8] and with $\lambda$ for the rectangular region $0<x<1 ;-1<y<1$. The value $\lambda_{1 / 3}\left(R_{2}\right)=13.737$ requires getting the least eigenvalue of a 7 th-order matrix, a relatively easy procedure with a desk machine.

The monotonicity of $\lambda_{h}\left(R_{2}\right)$ supports the author's conjecture ${ }^{2}$ that, for the $R$ of the theorem, $\lambda_{h}<\lambda$ for all $h$.

${ }^{2}$ See page 470 .

\section{REFERENCES}

1. N. Aronszajn and A. Zeichner, A neu type of auxiliary problem for approximation of eigenvalues by Weinstein's method, Technical Report 5, Oklahoma A. and M. College, Department of Mathematics, 1951.

2. Dorothy L. Bernstein, Existence theorems in partial differential equations, Annals of Mathematics Studies, No. 23, Princeton University Press, 1950.

3. Lothar Collatz, Eigenwertaufgaben mit technischen Anwendungen, Akademische Verlagsges., Leipzig, 1949.

4. Chandler Davis, Estimating eigenvalues, Proc. Amer. Math. Soc. 3 (1952), $942-947$.

5. George F. Forsythe, Asymptotic lower bounds for the fundamental frequency of convex membranes, multilithed typescript, National Bureau of Standards, Los Angeles, November 1953, 21 pp. (To appear in the Pacific Journal of Mathematics.)

6. L. V. Kantorovič and V.I. Krylov, Približennye metody vyš̌sego analiza (Approximate methods of higher analysis ), 3rd edit., Moscow-Leningrad, 1950.

7. Wilfred Kaplan, Advanced calculus, Addison-Wesley, Cambridge, 1952.

8. Tosio Kato, On the upper and lower bounds of eigenvalues, J. Phys. Soc. Japan 4 (1949), $334-339$.

9. William Edmund Milne, Numerical solution of differential equations, Wiley, New York and Chapman-Hall, London, 1953. 1952.

10. Zeev Nehari, Conformal mapping, McGraw-Hill, New York, Toronto, and London,

11. Georges Pólya, Sur une interprétation de la méthode des différences finies qui peut fournir des bornes supérieures ou inférieures, C. R. Acad. Sci. Paris 235 (1952), 995-997.

12. Estimates for eigenvalues, typescript, 1952. To appear in a von Mises "Festschrift." 
13. G. Pólya and G. Szegö, Isoperimetric inequalities in mathematical physics, Annals of Mathematics Studies, No. 27, Princeton University Press, 1951.

14. Mario G. Salvadori, Numerical computation of buckling loads by finite differences, Trans. Amer. Soc. Civil Engrs. 116 (1951), 590-636.

15. G. Temple, The computation of characteristic numbers and characteristic functions, Proc. London Math. Soc. (2) 29 (1929), 257-280.

16. , The accuracy of Rayleigh's method of calculating the natural frequencies of vibrating systems, Proc. Roy. Soc. London. Ser. A. 211 (1952), 204-224.

17. D. H. Weinstein, Modified Ritz method, Proc. Nat. Acad. Sci. U.S.A. 20 (1934), $529-532$.

18. Helmut Wieland, Die Einschliessung von Eigenwerten normaler Matrizen, Math. Ann. 121 (1949), 234-241.

NATIONAL BUREAU OF STANDARDS, LOS ANGELES

(Now with the University of California, Los Angeles) 


\section{PACIFIC JOURNAL OF MATHEMATICS}

\section{EDITORS}

\author{
M.M. SCHIFFE R* \\ Stanford University \\ Stanford, California \\ E. HEWITT \\ University of Washington \\ Seattle 5, Washington
}

R.P. DILWORTH

California Institute of Technology Pasadena 4, California

E.F. BECKENBACH**

University of California

Los Angeles 24, California

\section{ASSOCIATE EDITORS}

$\begin{array}{llll}\text { H. BUSEMANN } & \text { P.R. HALMOS } & \text { BORGE JESSEN } & \text { J. J. STOKER } \\ \text { HERBERT FEDERER } & \text { HEINZ HOPF } & \text { PAUL LÉVY } & \text { E.G. STRAUS } \\ \text { MARSHALL HALL } & \text { R.D. JAMES } & \text { GEORGE PÓLYA } & \text { KÔSAKU YOSIDA }\end{array}$

\section{SPONSORS}

UNIVERSITY OF BRITISH COLUMBIA CALIFORNIA INSTITUTE OF TECHNOLOGY UNIVERSITY OF CALIFORNIA, BERKELEY UNIVERSITY OF CALIFORNIA, DAVIS UNIVERSITY OF CALIFORNIA, LOS ANGELES UNIVERSITY OF CALIFORNIA, SANTA BARBARA UNIVERSITY OF NEVADA OREGON STATE COLLEGE UNIVERSITY OF OREGON
UNIVERSITY OF SOUTHERN CALIFORNIA STANFORD RESEARCH INSTITUTE STANFORD UNIVERSITY WASHINGTON STATE COLLEGE UNIVERSITY OF WASHINGTON

AMERICAN MATHEMATICAL SOCIETY HUGHES AIRCRAFT COMPANY

Mathematical papers intended for publication in the Pacific Journal of Mathematics should be typewritten (double spaced), and the author should keep a complete copy. Manuscripts may be sent to any of the editors. Manuscripts intended for the outgoing editors should be sent to their successors. All other communications to the editors should be addressed to the managing editor, E.G. Straus, at the University of California Los Angeles 24, California.

50 reprints of each article are furnished free of charge; additional copies may be obtained at cost in multiples of 50 .

The Pacific Journal of Mathematics is published quarterly, in March, June, September, and December. The price per volume (4 numbers) is $\$ 12.00$; single issues, $\$ 3.50$; back numbers (Volumes $1,2,3$ ) are available at $\$ 2.50$ per copy. Special price to individual faculty members of supporting institutions and to individual members of the American Mathematical Society: $\$ 4.00$ per volume; single issues, $\$ 1.25$.

Subscriptions, orders for back numbers, and changes of address should be sent to the publishers, University of California Press, Berkeley 4, California.

Printed at Ann Arbor, Michigan. Entered as second class matter at the Post Office, Berkeley, California.

* To be succeeded in 1955, by H.L. Royden, Stanford University, Stanford, California.

** To be succeeded in 1955, by E.G. Straus, University of California, Los Angeles 24, Calif.

UNIVERSITY OF CALIFORNIA PRESS - BERKELEY AND LOS ANGELES 


\section{Pacific Journal of Mathematics \\ Vol. 4, No. $3 \quad$ July, 1954}

Nelson Dunford, Spectral operators ........................ 321

John Wermer, Commuting spectral measures on Hilbert space.......... 355

Shizuo Kakutani, An example concerning uniform boundedness of spectral measures...................................... 363

William George Bade, Unbounded spectral operators .............. 373

William George Bade, Weak and strong limits of spectral operators ...... 393

Jacob T. Schwartz, Perturbations of spectral operators, and applications. I. Bounded perturbations ........................... 415

Mischa Cotlar, On a theorem of Beurling and Kaplansky............... 459

George E. Forsythe, Asymptotic lower bounds for the frequencies of certain polygonal membranes ............................... 467 